\title{
Autologous ACTR-CD16-CD28-expressing T-lymphocytes ACTR707
}

National Cancer Institute

\section{Source}

National Cancer Institute. Autologous ACTR-CD16-CD28-expressing T-lymphocytes

ACTR707. NCI Thesaurus. Code C139730.

A preparation of autologous T-lymphocytes that have been genetically modified, using proprietary Antibody-Coupled T-cell Receptor (ACTR) technology, to express a chimeric protein containing, at least, the extracellular Fc receptor domain of CD16, normally found on certain immune cells, such as natural killer (NK) cells, coupled to the co-stimulatory signaling domain of $C D 28$, with potential immunostimulating and antineoplastic activities. Upon reintroduction into the patient with co-administration of a cancer-specific antibody, the co-administered antibody targets and binds to the tumor-associated antigen (TAA) expressed on the tumor cell. In turn, the autologous ACT R-CD16-CD28-expressing Tlymphocytes ACT R707 bind to the antibody, become activated and induce the destruction of the tumor cells by a) releasing cytotoxins that directly kill cancer cells; b) releasing cytokines that trigger an immune response and recruit other immune-mediated killer cells to kill the tumor cells; c) targeting and killing adjacent tumor cells that are not bound to the antibody; d) inducing T-cell proliferation and thereby further enhancing the T-cell mediated tumor cell attack. Compared to other T-cell products, ACT R-based products do not target a specific TAA and can potentially be used in a variety of tumors because targeting is based on the specificity of the co-administered antibody. 